\title{
The microscopic origin of low thermal conductivity for enhanced thermoelectric performance of $\mathrm{Yb}$ doped $\mathrm{MgAgSb}$
}

Zihang Liu ${ }^{1,2}$, Yongsheng Zhang ${ }^{3,4}$, Jun $\mathrm{Mao}^{2,5}$, Weihong $\mathrm{Gao}^{6}$, Yumei Wang ${ }^{7}$, Jing Shuai $^{2}$, Wei Cai ${ }^{6}$, Jiehe Sui ${ }^{*}$, and Zhifeng Ren $^{2 *}$

${ }^{1}$ State Key Laboratory of Advanced Welding and Joining, Harbin Institute of Technology, Harbin 150001, China

${ }^{2}$ Department of Physics and TcSUH, University of Houston, Houston, Texas 77204, USA

${ }^{3}$ Key Laboratory of Materials Physics, Institute of Solid State Physics, Chinese Academy of Sciences, Hefei 230031, China

${ }^{4}$ University of Science and Technology of China, Hefei 230026, China

${ }^{5}$ Department of Mechanical Engineering, University of Houston, Houston, Texas 77204, USA

${ }^{6}$ National Key Laboratory for Precision Hot Processing of Metals and School of Materials Science and Engineering, Harbin Institute of Technology, Harbin 150001, China

${ }^{7}$ Beijing National Laboratory for Condensed Matter Physics, Institute of Physics, Chinese Academy of Sciences, P.O. Box 603, Beijing 100190, China

* To whom correspondence should be addressed. E-mail: suijiehe@hit.edu.cn, zren@uh.edu 


\section{Abstract}

An in-depth understanding of the origin of low lattice thermal conductivity $\kappa_{\text {lat }}$ is considered to be the prerequisite for developing and designing high thermoelectric performance materials. However, the microscopic mechanism of low $\kappa_{\text {lat }}$ for $\alpha-\mathrm{MgAgSb}$ has not been fully elaborated. Here we first thoroughly unveil the underlying mechanism using density functional theory (DFT) calculations. It is demonstrated that the unique lattice dynamic properties of $\alpha-\mathrm{MgAgSb}$ contribute to the low $\kappa_{\text {lat }}$, including a large number of optical branches, weak chemical bonding, the strong anharmonic coupling between longitudinal acoustic (LA) and longitudinal optical (LO) branches, high anharmonic behavior of LO branch. Nanostructuring has an effect in decreasing the lattice thermal conductivity due to the high density of boundaries and defects. Most importantly, $\mathrm{Yb}$ doping on the $\mathrm{Mg}$ site is chosen to optimize carrier concentration and simultaneously create strong point-defect phonon scattering. Collectively, a peak $Z T \sim 1.4$ at $550 \mathrm{~K}$ and average $Z T \sim 1.2$ from $300 \mathrm{~K}$ to $550 \mathrm{~K}$ have been achieved, which further demonstrates the realistic prospect of $\mathrm{MgAgSb}$ based thermoelectric materials for low-temperature waste heat harvesting.

Keywords: thermoelectric performance; DFT calculations; low lattice thermal conductivity; $\mathrm{MgAgSb}$; Yb doping

\section{Introduction}

To effectively harness energy for the huge global requirement, thermoelectric materials, capable of realizing the direct conversion from waste heat and solar energy into electricity, have received renewed interests in the past two decades [1-6]. But nowadays, thermoelectric devices are limited in niche markets because of the relatively low conversion efficiency, which is primarily dominated by the materials' 
thermoelectric figure of merit, $Z T=S^{2} \sigma \mathrm{T} / \kappa_{\text {total }}$, where $S, \sigma, T$, and $\kappa_{\text {total }}$ are the Seebeck coefficient, electrical conductivity, absolute temperature, and total thermal conductivity (including lattice thermal conductivity $\kappa_{\text {lat }}$ and electronic thermal conductivity $\kappa_{\text {ele }}$ ), respectively. Therefore, minimizing heat transport while simultaneously maintaining good electrical properties is the primary challenge for realizing high $Z T[7]$.

Due to the structural dependence of $\kappa_{\text {lat }}$ [3], alloying (or doping) and nanostructuring have been developed into effective strategies to suppress $\kappa_{\text {lat }}[8-15]$, even approaching to the amorphous limit. For instance, alloying (or doping) could create stable point-defect scattering centers for phonons with short mean free path [16], due to the mass fluctuation and surrounding strain field fluctuation. A larger difference about the atom mass and ionic radius between the host atom and alloying atom leads to the stronger phonon scattering, such as $\mathrm{Ba}$ doping in $\mathrm{BiCuSeO}$ and $\mathrm{Hf}$ alloying in $\mathrm{NbFeSb}[17,18]$. More importantly, proper alloying (or doping) could also tune carrier concentration $n$ near the optimized value to maximize the power factor $\left(P F=S^{2} \sigma\right)[19-21]$

Alternatively, searching for compounds possessing intrinsically low $\kappa_{\text {lat }}$ is critical for producing high thermoelectric performance. Those compounds usually exhibit some unique intrinsic features of crystal structure or physical properties, mainly including heavy atomic mass [22], complex unit cell [23], weak chemical bonding [24-26], liquid like ionic behavior and anharmonic bonding [27-34]. The representative example is SnSe crystals with ultralow $\kappa_{\text {lat }} \sim 0.6 \mathrm{~W} \mathrm{~m}^{-1} \mathrm{~K}^{-1}$ that exhibit the extremely weak $\mathrm{Sn}-\mathrm{Se}$ atomic interactions and large bond anharmonicity (Grüneisen parameter 3.1) [35]. Recently, the strong anharmonic coupling between transverse optical (TO) and longitudinal acoustic (LA) branches has been demonstrated to play a pronounced role in the low $\kappa_{\text {lat }}$ of PbTe using a combination of inelastic neutron scattering measurements and first-principles computations [36, 37]. 
$\alpha-\mathrm{MgAgSb}$ holds great promise for low temperature waste heat harvesting [38-45], primarily due to the intrinsically low $\kappa_{\text {lat }}$. However, the underlying microscopic mechanism of such a low $\kappa_{\text {lat }}$ has not been fully elaborated, especially in the lattice dynamics. Besides, because of the high optimal carrier concentration $\sim 9 \times 10^{19} \mathrm{~cm}^{-3}$ at $300 \mathrm{~K}$, previous efforts focus on tuning carrier concentration and thus enhancing $P F$ via doping with light atoms [38-40, 43, 46], such as Li and Na doping on $\mathrm{Mg}$ site $[39,43]$, but the final $Z T$ is almost unchanged due to the slightly decreased lattice thermal conductivity $[39,43]$. Since $\mathrm{Yb}$ belongs to the heavy-group rare-earth elements with a rather heavy atomic mass $\left(\sim 173.1 \mathrm{~g} \mathrm{~mol}^{-1}\right)$, thus it is highly expected that $\mathrm{Yb}$ doping could substantially suppress the lattice thermal conductivity due to the obviously decreased phonon group velocity and mean free path. Meanwhile, $\mathrm{Yb}$ has a comparable ionic radius $(\sim 93 \mathrm{pm})$ with that of $\mathrm{Mg}(\sim 65 \mathrm{pm})$, which may result in higher solid solubility on the $\mathrm{Mg}$ site in the distorted $\mathrm{MgAgSb}$ lattice. Besides, $\mathrm{YbAgSb}$ alloy exhibits a typical metal behavior with a low electrical resistivity $\left(\sim 1.5 \times 10^{-6} \Omega \mathrm{m}\right.$ at room temperature) [47], so $\mathrm{Yb}$ doping (or alloying) could also reduce the electrical resistivity. Based on the above considerations, it is highly expected that rationally designed Yb doping not only observably suppresses $\kappa_{\text {lat }}$ but also simultaneously optimizes electrical properties to achieve high ZT.

Herein, we thoroughly explore the microscopic origin of intrinsically low $\kappa_{\text {lat }}$ of $\alpha-\mathrm{MgAgSb}$ in the lattice dynamics using density functional theory (DFT) calculations. Microstructural observations also indicate that high density of boundaries and defects could further decrease the lattice thermal conductivity. More importantly, we achieve a peak $Z T \sim 1.4$ and average $Z T \sim 1.2$ via $\mathrm{Yb}$ doping, originated from tuning carrier concentration and simultaneously creating strong phonon-scattering. Assuming a leg length of $2 \mathrm{~mm}$, cold side temperature of $323 \mathrm{~K}$ and hot side temperature of $548 \mathrm{~K}$, the highest calculated conversion efficiency is around 9.4\%, higher than undoped (8.4\%), Li doped (8.6\%) and the measured efficiency of a single leg based on $\mathrm{Ni}$ 
doped $\mathrm{MgAgSb}(8.5 \%$ between $293 \mathrm{~K}$ and $518 \mathrm{~K})$ [38, 43].

\section{Experimental Section}

Synthesis. Mg (99.98\%; Alfa Aesar), Ag (99.99\%; Sigma Aldrich), Sb (99.999\%; Alfa Aesar) and Yb (99.95\%; Alfa Aesar) were weighed according to the nominal composition $\mathrm{Mg}_{1-\mathrm{x}} \mathrm{Yb}_{\mathrm{x}} \mathrm{Ag}_{0.97} \mathrm{Sb}_{0.99}$ samples ( $\mathrm{x}=0,0.0025,0.05$ and 0.01 ) and then loaded into the stainless steel jar in a glove-box under argon atmosphere. The two-step ball milling process was identical to our previous reports and finally the ball-milled powder was loaded into graphite die and hot pressed at $573 \mathrm{~K}$ for $5 \mathrm{~min}$ under a pressure of $80 \mathrm{MPa}$.

X-ray diffraction. X-ray diffraction (XRD) analysis was performed using a PANa-lytical multipurpose diffractometer with an X'celerator detector (PANalyticalX'Pert Pro). The phases were analyzed with JADE 6.0 software. The diffraction peaks of all the hot pressed samples exhibit excellent match with the $\alpha-\operatorname{MgAgSb}$ (space group $I \overline{4} C 2$ ) without any impurity phase within the detection limit of the XRD spectrometer [48], shown in the Figure S1.

Physical Characterization. Bar samples were cut from the disks and used for simultaneous measurement of the electrical resistivity $(\rho)$ and Seebeck coefficient $(S)$ on a commercial system (ULVAC ZEM-3). The thermal conductivity was calculated using $\kappa=D C_{p} d$, where $D, C_{p}$, and $d$ are the thermal diffusivity, specific heat capacity, and density, respectively. The thermal diffusivity coefficient $(D)$ was measured using the coin sample on a laser flash system (Netzsch LFA 457, Germany). The specific heat capacity $\left(C_{p}\right)$ was measured on a differential scanning calorimetry thermal analyzer (Netzsch DSC $404 \mathrm{C}$, Germany). The density $(d)$ around $6.2 \mathrm{~g} \mathrm{~cm}^{-3}$ was determined by the Archimedes method. The Hall Coefficient $R_{H}$ at room temperature was measured using the PPMS (Physical Properties Measurement System, Quantum Design). The Hall carrier concentration $\left(n_{H}\right)$ was obtained by $n_{H}=1 / e R_{H}$ and the Hall 
carrier mobility $\left(\mu_{\mathrm{H}}\right)$ was calculated by $\sigma=e \mu_{H} n_{H}$, where $e$ is the electronic charge and $\sigma$ the electrical conductivity.

Theoretical calculation. DFT calculations are performed using the Vienna $\mathrm{Ab}$ initio Simulation Package (VASP) with the projector augmented wave (PAW) scheme [49], and the generalized gradient approximation of Perdew, Burke and Ernzerhof (GGA-PBE) for the electronic exchange-correlation functional [50]. The energy cut-off for the plane wave expansion is $500 \mathrm{eV}$. The Brillouin zones are sampled by Monkhorst-Pack k-point meshes meshes for all compounds [51], with meshes chosen to give a roughly constant density of $\mathrm{k}$ points $\left(30 \mathrm{~A}^{\circ 3}\right)$. Atomic positions and unit cell vectors are relaxed until all the forces and components of the stress tensor are below $0.01 \mathrm{eV} / \mathrm{A}^{\circ}$ and $0.2 \mathrm{kbar}$, respectively. Vibrational properties are calculated using the supercell (192 atoms in the $2 \times 2 \times 2$ supercell) force constant method by the Phononpy [52]. In the quasiharmonic DFT phonon calculations, the system volume is isotropically expanded by $4 \%$ from the DFT relaxed volume. The Grüneisen parameter $\left(\gamma_{i}\right)$ is defined as $[30,53]$

$$
\gamma_{i}=-\frac{V}{\omega_{i}} \frac{\partial \omega_{i}}{\partial V}
$$

It characterizes the relationship between phonon frequency and volume change. The Debye temperature $\left(\theta_{D}\right)$ is calculated using $\theta_{D}=\omega_{D} / k_{B}$, where $\omega_{D}$ is the largest acoustic frequency in each direction; the phonon velocity $v$ is the slope of the acoustic phonon dispersion around the point. The Grüneisen parameters, Debye temperatures, and phonon velocities are averaged by the weight of the high-symmetry points.

\section{Results and discussion}

\section{Synergistic optimization of thermoelectric performance}

Figure 1 shows the synergistic strategies of enhancing thermoelectric performance via $\mathrm{Yb}$ doping and the corresponding $Z T$ as well as calculated efficiency. 
First, $\mathrm{Yb}$ doping tunes the Fermi level closer to the valence band and thus enhances $P F$ (Figure 1a). Second, the intrinsically low $\kappa_{\text {lat }}$ of $\mathrm{MgAgSb}$ contributes to the high $Z T$, which can be attributed to the unique lattice dynamic properties, including large number of optical phonon branches, weak chemical bonding, longitudinal optical (LO)-longitudinal acoustic (LA) branches scattering and high anharmonic behavior of LO branch (Figure 1b). Third, Yb doping could further suppress $\kappa_{\text {lat }}$ because of the strong point-defect scattering to phonons due to the large difference in atom mass and size between $\mathrm{Mg}$ and $\mathrm{Yb}$ (Figure 1c). Due to the enhanced $P F$ and significantly suppressed $\kappa_{\text {lat }}$, Figure 1d shows that $Z T$ has been improved over the whole measured temperature range upon $\mathrm{Yb}$ doping. Specifically, $\mathrm{Mg}_{0.995} \mathrm{Yb}_{0.005} \mathrm{Ag}_{0.97} \mathrm{Sb}_{0.99}$ exhibits the highest average $Z T \sim 1.2$, higher than that of $\operatorname{MgAg}_{0.97} \mathrm{Sb}_{0.99} \sim 1.0$ and $\mathrm{Mg}_{0.99} \mathrm{Li}_{0.01} \mathrm{Ag}_{0.97} \mathrm{Sb}_{0.99} \sim 1.1$ (Figure 1e) [42, 43]. According to the method proposed by Kim et al. and assuming a leg length of $2 \mathrm{~mm}$, cold side temperature of $323 \mathrm{~K}$ and hot side temperature of $548 \mathrm{~K}$ (see the supporting information) [54], Figure 1e shows that the corresponding calculated single leg conversion efficiency is around $9.4 \%$, higher than undoped $(8.4 \%)$, Li doped $(8.6 \%)$ as well as the measured efficiency of a single leg based on Ni doped MgAgSb (8.5\% between $293 \mathrm{~K}$ and $518 \mathrm{~K})$ [38, 43].

\section{Enhancing power factor by tuning carrier concentration}

Figure 2 presents the electrical transport properties of $\mathrm{Mg}_{1-\mathrm{x}} \mathrm{Yb}_{\mathrm{x}} \mathrm{Ag}_{0.97} \mathrm{Sb}_{0.99}$. Hall carrier concentration $n$ first increases and then saturates, which may be related with low solid solubility of $\mathrm{Yb}$ on the $\mathrm{Mg}$ sublattice, while mobility $\mu$ monotonically decreases with increasing $\mathrm{Yb}$ doping concentration. Electrical resistivity $\rho$ first decreases and then arises with the increased doping concentration (Figure 2b). Since $\mathrm{YbAgSb}$ exhibits a typical metal behavior (room-temperature $\rho \sim 1.5 \times 10^{-6} \Omega \mathrm{m}$ ) [47], $\mathrm{Yb}$ doping on the $\mathrm{Mg}$ site leads to reduced $\rho$ compared with pristine $\mathrm{Mg} \mathrm{Ag}_{0.97} \mathrm{Sb}_{0.99}$. In addition, $\rho$ of undoped and doped samples fit the temperature dependence of $T^{3 / 2}$ 
before the intrinsic excitation, indicating carrier transport mechanism is mainly dominated by acoustic phonon scattering at the low temperature range. Figure $2 \mathrm{c}$ shows that Seebeck coefficient $S$ decreases somewhat upon $\mathrm{Yb}$ doping, due to the increased $n$. Figure 2d shows the Pisarenko plots, $S$ dependence on $n$, with $m^{*}=2.4$ $\mathrm{m}_{0}$ at room temperature assuming single parabolic band model and the acoustic phonon scattering mechanism $[43,55]$, where $\mathrm{m}_{0}$ is the electron mass. Apparently, all the experimental data, including $\mathrm{Yb}$ and $\mathrm{Li}$ doping and tuning of Sb content $[42,43]$, fall nearly on the calculated line, indicating that the valence band structure remains almost unchanged. Compared with pristine $\mathrm{MgAg}_{0.97} \mathrm{Sb}_{0.99}, P F$ could be finally enhanced by around $20 \%$ at the entire temperature range for $\mathrm{Mg}_{0.995} \mathrm{Yb}_{0.005} \mathrm{Ag}_{0.97} \mathrm{Sb}_{0.99}$ (Figure 2e). Since the band structure is almost unchanged upon $\mathrm{Yb}$ doping, tuning carrier concentration should be the only approach to enhance $P F$. As aforementioned, $\mathrm{Yb}$ doping increases the carrier concentration from $2.9 \times 10^{19} \mathrm{~cm}^{-3}\left(\mathrm{MgAg}_{0.97} \mathrm{Sb}_{0.99}\right)$ to $4.6 \times 10^{19} \mathrm{~cm}^{-3}\left(\mathrm{Mg}_{0.995} \mathrm{Yb}_{0.005} \mathrm{Ag}_{0.97} \mathrm{Sb}_{0.99}\right)$, meanwhile shifting the Fermi level from -0.75 to -0.13 (Table 1). Normally, the optimal reduced Fermi energy $\eta$ should be close to zero for most thermoelectric materials with an acoustic phonon scattering mechanism for charge carrier [56]. This statement can well explain the enhancement of $P F$ upon $\mathrm{Yb}$ doping. Nevertheless, the optimized carrier concentration $n_{\text {opt }}$ is around $9 \times 10^{19} \mathrm{~cm}^{-3}$ (Figure 2f) [43], still higher than that of $\mathrm{Yb}$ doping. Further enhancement of $P F$ could be possible by dual doping.

\section{Suppressing lattice thermal conductivity via strong point-defect scattering}

Figure $3 \mathrm{a}$ shows that total thermal conductivity $\kappa_{\text {total }}$ first decreases because of Umklapp scattering and then increases significantly due to bipolar effect. Electrical thermal conductivity $\kappa_{\text {ele }}$ can be calculated from the Wiedemann-Franz relationship $\left(\kappa_{\text {ele }}=L \sigma T\right)$, where $L$ is the Lorenz number calculated based on the single parabolic band model with the acoustic phonon scattering mechanism [43, 55]. Thus, $\kappa_{\text {lat }}$ is obtained by subtracting $\kappa_{\text {ele }}$ from $\kappa_{\text {total }}$ before intrinsic excitation, shown in the Figure 
3b. It is obvious that the pristine $\mathrm{MgAg}_{0.97} \mathrm{Sb}_{0.99}$ has a low $\kappa_{\text {lat }}$, room-temperature value only around $0.66 \mathrm{~W} \mathrm{~m}^{-1} \mathrm{~K}^{-1}$, which is mainly due to the intrinsic lattice dynamics properties that will be thoroughly discussed latter. Upon $\mathrm{Yb}$ doping, room-temperature $\kappa_{\text {lat }}$ decreases to $0.6 \mathrm{~W} \mathrm{~m}^{-1} \mathrm{~K}^{-1}$ for $\mathrm{Mg}_{0.995} \mathrm{Yb}_{0.005} \mathrm{Ag}_{0.97} \mathrm{Sb}_{0.99}$. Generally, materials with low $\kappa_{\text {lat }}$ possess short phonon mean free path (MFP) $l$ that can be evaluated by the following equation:

$$
k_{\text {lat }}=\frac{1}{3} C_{v} v_{a} l
$$

where $C_{v}$ is the heat capacity, $v_{a}$ is the average sound velocity $\sim 1276 \mathrm{~m} \mathrm{~s}^{-1}$. The obtained phonon mean free path $l$ of nanostructured $\mathrm{MgAg}_{0.97} \mathrm{Sb}_{0.99}$ is around $0.53 \mathrm{~nm}$, even less than that of $\mathrm{SnSe}(0.84 \mathrm{~nm})$ [35]. This points out that phonon scattering by point defect is an effective strategy to further reduce the $\kappa_{\text {lat }}$. Yb doping will generate strong phonon scattering due to the large mass and size difference (Mg: $24.3 \mathrm{~g} \mathrm{~mol}^{-1}$ and $66 \mathrm{pm}$ vs. Yb: $173.1 \mathrm{~g} \mathrm{~mol}^{-1}$ and $93 \mathrm{pm}$ ) causing surrounding strain field fluctuation based on the Rayleigh-type scattering mechanism [57]. Since $\mathrm{Mg}_{0.995} \mathrm{Yb}_{0.005} \mathrm{Ag}_{0.97} \mathrm{Sb}_{0.99}$ has the comparable lattice thermal conductivity with that of $\mathrm{Mg}_{0.99} \mathrm{Li}_{0.01} \mathrm{Ag}_{0.97} \mathrm{Sb}_{0.99}$ [43], it is reasonable to say that $\mathrm{Yb}$ doping is more effective in suppressing lattice thermal conductivity. Due to that $\mathrm{Li}\left(6.94 \mathrm{~g} \mathrm{~mol}^{-1}, 60 \mathrm{pm}\right)$ has a smaller difference between atomic mass and ionic radius with $\mathrm{Mg}, \mathrm{Li}$ has a larger solid solubility than $\mathrm{Yb}$ on the $\mathrm{Mg}$ site, which could result in the higher carrier concentration and power factor in comparison with $\mathrm{Yb}$ doping. However, $\mathrm{Yb}$ doping has more obvious effect in suppressing lattice thermal conductivity. The modified Debye-Callaway model is employed to semi-qualitatively compare the contribution of site disorder in the lattice $[43,58,59]$, presented in the supporting information. The calculated results, given as dash lines in Figure $3 b$, show good agreement with experimental values before the onset of bipolar effect. Table 2 shows the calculation parameters, where parameter A corresponds to the strength of phonon scattering of point defects. The gradually increased parameter A indicates stronger and stronger 
point-defect scattering upon $\mathrm{Yb}$ doping, consistent with previous analysis.

\section{Exploring the origin of low lattice thermal conductivity}

Since low $\kappa_{\text {lat }}$ in solid has close relation with the lattice dynamics properties, phonon and Grüneisen dispersions along the high symmetry directions within the Brillouin zone are calculated using DFT phonon calculations within the quasi-harmonic approximation (Figure 4) [49-52]. Because of the large number of atoms (24 atoms) in the primitive cell of $\mathrm{MgAgSb}$, there will be 3 acoustic branches and 69 optical branches. It is generally assumed that optical branches have small or negligible contributions to $\kappa_{\text {lat }}$ in bulk materials mainly due to their low phonon velocities [60], which is beneficial to achieve low $\kappa_{\text {lat }}[61]$. Figure $4 \mathrm{a}$ shows one extremely anomalous longitudinal optical (LO) branch (orange). The boundary frequency of LO branch at the $\Gamma$ point is extremely low $\left(\sim 17 \mathrm{~cm}^{-1}\right)$, even smaller than that of $\operatorname{SnSe}\left(\sim 20 \mathrm{~cm}^{-1}\right)$ and $\mathrm{PbTe}\left(\sim 32 \mathrm{~cm}^{-1}\right)[33,37]$, indicating the soft nature of this LO branch. It has a large slope of the phonon dispersion around the $\Gamma$ point that means a high phonon velocity and thus may partially participate in heat transporting, similar with that of $\mathrm{PbTe}[37,62]$. In addition, there is a strong volume dependence of the LO phonon frequency around the zone center that implies the strong anharmonic behavior of this LO branch. More importantly, the observed "avoided crossing" interaction between LO and longitudinal acoustic (LA) branches leads to the abruptly changed slope away from the center of Brillouin zone [36, 63], especially for LA branch along the $\Gamma$-Y direction where the acoustic character is maintained only within a small region of wave vectors with $|q|<0.2 \AA^{-1}$. However, it is rather difficult to qualitatively calculate the contribution of this LO branch, due to this extremely time-consuming feature, for example using ShengBTE to solve the Boltzmman Transport Theory [64]. For those significant heat transport acoustic phonon branches, the saturated frequencies merely reach around $20-35 \mathrm{~cm}^{-1}$, which are even lower than 
those of $\mathrm{SnSe}$ and $\mathrm{PbTe}[33,37]$. Hence, those anomalously low acoustic-mode frequencies means the intrinsically weak chemical bonding in $\mathrm{MgAgSb}$ compound, which could be directly manifested by the low Young's modulus of $\mathrm{MgAgSb}$ (55.0 GPa) [65]. The weak chemical bonding results in the phonon modes softening that finally mitigates phonon propagation. The weak chemical bonding is possibly related with Ag-Sb bonding, where Ag has an abnormal occupied site, which leads to the low defect formation energy of $\mathrm{Ag}$ vacancy in $\alpha-\mathrm{MgAgSb}$ revealed by first-principles calculation [44].

To quantitatively elucidate the strength of anharmonicity of $\mathrm{MgAgSb}$, the dispersion of the Grüneisen parameters for all acoustic branches and special LO branch are calculated. According to the formula proposed by Leibfried and Schlömann [66], the lattice thermal conductivity $\kappa_{\text {lat }}$ is given by:

$$
\kappa_{l a t}=B \frac{M V^{1 / 3} \theta_{D}^{3}}{\gamma^{2} T}
$$

where $B$ a numerical coefficient, $M$ the average atomic mass, $V$ the average atomic volume and $\Theta_{D}$ the Debye temperature. As known, anharmonic bonding could strengthen phonon-phonon interaction and thus significantly suppress the lattice thermal conductivity [30]. As shown in Figure 4b, the majority of Grüneisen parameters along the high symmetry directions are positive throughout the Brillouin zone. Both LA and LO branches possess higher Grüneisen parameters, compared with those of two TA branches. Typically, the maximum Grüneisen parameters of LO branch around $\Gamma$ point is extraordinarily high $\sim 5.8$. The average corresponding Grüneisen parameters $(\bar{\gamma})$ of three acoustic branches are calculated based on $\bar{\gamma}=\sqrt{\left(\gamma_{i}\right)^{2}}$, shown in Table 2. The $\bar{\gamma}$ of LA branch is about 1.9, surpassing that of TA and $\mathrm{TA}^{\prime}$ branches by a factor of at least 2 . The anharmonic behavior may be related with the distorted $\mathrm{Mg}-\mathrm{Sb}$ rocksalt lattice [48]. Besides, we can clearly observe the sudden change of Grüneisen parameters around $\Gamma$ point for LA and LO branches, 
originated from the "avoided-crossing" interaction [36, 63].

Based on the phonon dispersion, the Debye temperatures and phonon velocities of three acoustic phonon branches can be also obtained, shown in Table 3. Those three acoustic branches (TA, TA', and LA) have an extremely low Debye temperature (39 K, $44 \mathrm{~K}, 49 \mathrm{~K})$, even lower than that of $\mathrm{SnSe}(62 \mathrm{~K}, 72 \mathrm{~K}, 62 \mathrm{~K})$ [33]. Since there is a close relationship between the stiffness and Debye temperature, the low Debye temperatures of those three acoustic branches means the weak chemical bonding. Similarly, the low phonon velocities could demonstrate this conclusion as well. Due to that our DFT phonon calculation is based on the crystal structure of undoped $\mathrm{MgAgSb}$, it could not give any information about the effect of $\mathrm{Yb}$ doping on phonon transport. In addition, it is impossible to perform the phonon calculation about $\mathrm{Yb}$ doping, because the supercell would become extremely large because of the very low $\mathrm{Yb}$ doping content in our work. Except for this unique lattice dynamic properties of pristine $\mathrm{MgAgSb}$, nanostructuring has been demonstrated as an effective strategy to further suppress $\kappa_{\text {lat }}$. As reported by Ying et al. [45], pristine MgAgSb with coarse grains has a room-temperature $\kappa_{\text {lat }}$ around $0.82 \mathrm{~W} \mathrm{~m}^{-1} \mathrm{~K}^{-1}, 24 \%$ higher than that of nanostructured $\mathrm{MgAgSb}\left(0.66 \mathrm{~W} \mathrm{~m}^{-1} \mathrm{~K}^{-1}\right)$. This suppression could be ascribed to the strong phonon scattering through high density of boundaries and defects mainly resulted from long-time high energy ball milling process [43]. Here, we perform an investigation on the microstructure of $\mathrm{Mg}_{0.995} \mathrm{Yb}_{0.005} \mathrm{Ag}_{0.97} \mathrm{Sb}_{0.99}$ by means of scanning electron microscope (SEM) and transmission electron microscope (TEM), given in the Figure 5. The results clearly show that nanostructured $\mathrm{MgAgSb}$ has an average grain size around $150 \mathrm{~nm}$ as well as high density of stacking faults and dislocations, which could scatter medium and long-wavelength phonons.

\section{Conclusion}

In summary, DFT calculations demonstrate that the unique lattice dynamic properties 
contribute to the low $\kappa_{\text {lat }}$ of $\mathrm{MgAgSb}$, including a large number of optical branches, weak chemical bonding, strong anharmonic coupling between LO and LA branches, and high anharmonic behavior of LO branch. In addition, nanostructuring has an effect in decreasing the lattice thermal conductivity due to the high density of boundaries and defects. Furthermore, Yb doping continues to obviously decrease the $\kappa_{\text {lat }}$ because of the strong point-defect phonon scattering. Simultaneously, tuning the carrier concentration via $\mathrm{Yb}$ doping also leads to enhanced $P F$ around $20 \%$. As a result, a peak $Z T \sim 1.4$ and average $Z T \sim 1.2$ have been achieved, which highlights the promising prospect of $\mathrm{Yb}$ doped $\mathrm{MgAgSb}$ materials for low temperature power generation.

\section{Acknowledgement}

This work was supported by "Solid State Solar Thermal Energy Conversion Center ( $S^{3}$ TEC)", an Energy Frontier Research Center funded by the U.S. Department of Energy, Office of Science, Office of Basic Energy Science under award number DE-SC0001299; and was also supported by National Natural Science Foundation of China (no. 51471061 and 51271069) (Calculation part). Y. Z acknowledges financial support from National Natural Science Foundation of China No. 11474283 and The Major/Innovative Program of Development Foundation of Hefei Center for Physical Science and Technology No. 2014FXCX001. 


\section{References}

[1] D.M. Rowe, CRC handbook of thermoelectrics, CRC press, 1995.

[2] M.S. Dresselhaus, G. Chen, M.Y. Tang, R. Yang, H. Lee, D. Wang, Z. Ren, J.P. Fleurial, P. Gogna, New Directions for Low-Dimensional Thermoelectric Materials, Advanced Materials 19(8) (2007) 1043-1053.

[3] G.J. Snyder, E.S. Toberer, Complex thermoelectric materials, Nature Materials 7(2) (2008) 105-114.

[4] W. Liu, X. Yan, G. Chen, Z. Ren, Recent advances in thermoelectric nanocomposites, Nano Energy 1(1) (2012) 42-56.

[5] X. Shi, L. Chen, C. Uher, Recent advances in high-performance bulk thermoelectric materials, International Materials Reviews 61 (2016) 379.

[6] J. Mao, Z. Liu, Z. Ren, Size effect in thermoelectric materials, npj Quantum Materials 1 (2016) 16028

[7] J. Yang, L. Xi, W. Qiu, L. Wu, X. Shi, L. Chen, J. Yang, W. Zhang, C. Uher, D.J. Singh, On the tuning of electrical and thermal transport in thermoelectrics: an integrated theory-experiment perspective, npj Computational Materials 2 (2016) 15015.

[8] J. Callaway, H.C. von Baeyer, Effect of point imperfections on lattice thermal conductivity, Physical Review 120(4) (1960) 1149.

[9] Q. Shen, L. Chen, T. Goto, T. Hirai, J. Yang, G.P. Meisner, C. Uher, Effects of partial substitution of $\mathrm{Ni}$ by Pd on the thermoelectric properties of ZrNiSn-based half-Heusler compounds, Applied Physics Letters 79(25) (2001) 4165-4167.

[10] H. Xie, H. Wang, Y. Pei, C. Fu, X. Liu, G.J. Snyder, X. Zhao, T. Zhu, Beneficial Contribution of Alloy Disorder to Electron and Phonon Transport in Half Heusler Thermoelectric Materials, Advanced Functional Materials 23(41) (2013) 5123-5130.

[11] J. Mao, Y. Wang, B. Ge, Q. Jie, Z. Liu, U. Saparamadu, W. Liu, Z. Ren, Thermoelectric performance enhancement of $\mathrm{Mg}_{2} \mathrm{Sn}$ based solid solutions by band convergence and phonon scattering via $\mathrm{Pb}$ and $\mathrm{Si} / \mathrm{Ge}$ substitution for $\mathrm{Sn}$, Physical Chemistry Chemical Physics 18(30) (2016) 20726-20737.

[12] B. Poudel, Q. Hao, Y. Ma, Y. Lan, A. Minnich, B. Yu, X. Yan, D. Wang, A. Muto, D. Vashaee, High-thermoelectric performance of nanostructured bismuth antimony telluride bulk alloys, Science 320(5876) (2008) 634-638.

[13] Y. Lan, A.J. Minnich, G. Chen, Z. Ren, Enhancement of Thermoelectric Figure of - Merit by a Bulk Nanostructuring Approach, Advanced Functional Materials 20(3) (2010) 357-376.

[14] J.-F. Li, W.-S. Liu, L.-D. Zhao, M. Zhou, High-performance nanostructured thermoelectric materials, NPG Asia Materials 2(4) (2010) 152-158.

[15] Z. Liu, Y. Pei, H. Geng, J. Zhou, X. Meng, W. Cai, W. Liu, J. Sui, Enhanced thermoelectric performance of $\mathrm{Bi}_{2} \mathrm{~S}_{3}$ by synergistical action of bromine substitution and copper nanoparticles, Nano Energy 13 (2015) 554-562.

[16] C. Walker, R. Pohl, Phonon scattering by point defects, Physical Review 131(4) (1963) 1433.

[17] J. Li, J. Sui, Y. Pei, C. Barreteau, D. Berardan, N. Dragoe, W. Cai, J. He, L.-D. Zhao, A high thermoelectric figure of merit ZT> 1 in Ba heavily doped $\mathrm{BiCuSeO}$ oxyselenides, Energy \& Environmental Science 5(9) (2012) 8543-8547. 
[18] C. Fu, S. Bai, Y. Liu, Y. Tang, L. Chen, X. Zhao, T. Zhu, Realizing high figure of merit in heavy-band p-type half-Heusler thermoelectric materials, Nature Communications 6 (2015).

[19] Y. Pei, A. LaLonde, S. Iwanaga, G.J. Snyder, High thermoelectric figure of merit in heavy hole dominated PbTe, Energy \& Environmental Science 4(6) (2011) 2085-2089.

[20] Y. Pei, H. Wang, G. Snyder, Band engineering of thermoelectric materials, Advanced Materials 24(46) (2012) 6125-6135.

[21] Z. Liu, H. Geng, J. Shuai, Z. Wang, J. Mao, D. Wang, Q. Jie, W. Cai, J. Sui, Z. Ren, The effect of nickel doping on electron and phonon transport in the n-type nanostructured thermoelectric material CoSbS, Journal of Materials Chemistry C 3(40) (2015) 10442-10450.

[22] S.R. Brown, S.M. Kauzlarich, F. Gascoin, G.J. Snyder, Yb14MnSb11: New high efficiency thermoelectric material for power generation, Chemistry of materials 18(7) (2006) 1873-1877.

[23] K. Kurosaki, A. Kosuga, H. Muta, M. Uno, S. Yamanaka, Ag9TlTe5: a high-performance thermoelectric bulk material with extremely low thermal conductivity, Applied Physics Letters 87(6) (2005).

[24] Y.-L. Pei, J. He, J.-F. Li, F. Li, Q. Liu, W. Pan, C. Barreteau, D. Berardan, N. Dragoe, L.-D. Zhao, High thermoelectric performance of oxyselenides: intrinsically low thermal conductivity of Ca-doped $\mathrm{BiCuSeO}$, NPG Asia Materials 5(5) (2013) e47.

[25] W. Qiu, L. Xi, P. Wei, X. Ke, J. Yang, W. Zhang, Part-crystalline part-liquid state and rattling-like thermal damping in materials with chemical-bond hierarchy, Proceedings of the National Academy of Sciences 111(42) (2014) 15031-15035.

[26] S. Wang, Y. Sun, J. Yang, B. Duan, L. Wu, W. Zhang, J. Yang, High thermoelectric performance in Te-free $(\mathrm{Bi}, \mathrm{Sb})_{2} \mathrm{Se}_{3}$ via structural transition induced band convergence and chemical bond softening, Energy \& Environmental Science 9 (2016) 3436-3447.

[27] H. Liu, X. Shi, F. Xu, L. Zhang, W. Zhang, L. Chen, Q. Li, C. Uher, T. Day, G.J. Snyder, Copper ion liquid-like thermoelectrics, Nature Materials 11(5) (2012) 422-425.

[28] Y. He, T. Day, T. Zhang, H. Liu, X. Shi, L. Chen, G.J. Snyder, High Thermoelectric Performance in Non-Toxic Earth-Abundant Copper Sulfide, Advanced Materials 26(23) (2014) 3974-3978.

[29] E.J. Skoug, D.T. Morelli, Role of lone-pair electrons in producing minimum thermal conductivity in nitrogen-group chalcogenide compounds, Physical Review Letters 107(23) (2011) 235901.

[30] Y. Zhang, E. Skoug, J. Cain, V. Ozoliņš, D. Morelli, C. Wolverton, First-principles description of anomalously low lattice thermal conductivity in thermoelectric $\mathrm{Cu}-\mathrm{Sb}-\mathrm{Se}$ ternary semiconductors, Physical Review B 85(5) (2012) 054306.

[31] M.D. Nielsen, V. Ozolins, J.P. Heremans, Lone pair electrons minimize lattice thermal conductivity, Energy \& Environmental Science 6(2) (2013) 570-578.

[32] Y. Zhang, V. Ozolinšs, D. Morelli, C. Wolverton, Prediction of New Stable Compounds and Promising Thermoelectrics in the $\mathrm{Cu}-\mathrm{Sb}-\mathrm{Se}$ System, Chemistry of Materials 26(11) (2014) 3427-3435.

[33] L.-D. Zhao, S.-H. Lo, Y. Zhang, H. Sun, G. Tan, C. Uher, C. Wolverton, V.P. Dravid, M.G. Kanatzidis, Ultralow thermal conductivity and high thermoelectric 
figure of merit in SnSe crystals, Nature 508(7496) (2014) 373-377.

[34] S. Lee, K. Esfarjani, T. Luo, J. Zhou, Z. Tian, G. Chen, Resonant bonding leads to low lattice thermal conductivity, Nature Communications 5(4) (2014) 3525-3525.

[35] Y. Xiao, C. Chang, Y. Pei, D. Wu, K. Peng, X. Zhou, S. Gong, J. He, Y. Zhang, Z. Zeng, Origin of low thermal conductivity in SnSe, Physical Review B 94(12) (2016) 125203.

[36] O. Delaire, J. Ma, K. Marty, A.F. May, M.A. McGuire, M.-H. Du, D.J. Singh, A. Podlesnyak, G. Ehlers, M. Lumsden, Giant anharmonic phonon scattering in PbTe, Nature Materials 10(8) (2011) 614-619.

[37] Z. Tian, J. Garg, K. Esfarjani, T. Shiga, J. Shiomi, G. Chen, Phonon conduction in $\mathrm{PbSe}, \mathrm{PbTe}$, and $\mathrm{PbTe}_{1-\mathrm{x}} \mathrm{Se}_{\mathrm{x}}$ from first-principles calculations, Physical Review B 85(18) (2012) 184303.

[38] H. Zhao, J. Sui, Z. Tang, Y. Lan, Q. Jie, D. Kraemer, K. McEnaney, A. Guloy, G. Chen, Z. Ren, High thermoelectric performance of MgAgSb-based materials, Nano Energy 7 (2014) 97-103.

[39] J. Shuai, H.S. Kim, Y. Lan, S. Chen, Y. Liu, H. Zhao, J. Sui, Z. Ren, Study on thermoelectric performance by $\mathrm{Na}$ doping in nanostructured $\mathrm{Mg}_{1-\mathrm{x}} \mathrm{Na}_{\mathrm{x}} \mathrm{Ag}_{0.97}$ $\mathrm{Sb}_{0.99}$, Nano Energy 11 (2015) 640-646.

[40] J. Sui, J. Shuai, Y. Lan, Y. Liu, R. He, D. Wang, Q. Jie, Z. Ren, Effect of Cu concentration on thermoelectric properties of nanostructured p-type $\mathrm{MgAg}_{0.97-}$ ${ }_{\mathrm{x}} \mathrm{Cu}_{\mathrm{x}} \mathrm{Sb}_{0.99}$, Acta Materialia 87 (2015) 266-272.

[41] D. Kraemer, J. Sui, K. McEnaney, H. Zhao, Q. Jie, Z. Ren, G. Chen, High thermoelectric conversion efficiency of $\mathrm{MgAgSb}$-based material with hot-pressed contacts, Energy \& Environmental Science 8(4) (2015) 1299-1308.

[42] Z. Liu, J. Shuai, J. Mao, Y. Wang, Z. Wang, W. Cai, J. Sui, Z. Ren, Effects of antimony content in $\mathrm{MgAg}_{0.97} \mathrm{Sb}_{\mathrm{x}}$ on output power and energy conversion efficiency, Acta Materialia 102 (2016) 17-23.

[43] Z. Liu, Y. Wang, J. Mao, H. Geng, J. Shuai, Y. Wang, R. He, W. Cai, J. Sui, Z. Ren, Lithium doping to enhance thermoelectric performance of MgAgSb with weak electron-phonon coupling, Advanced Energy Materials 6(7) (2016) 1502269 .

[44] Z. Liu, H. Geng, J. Mao, S. Jing, R. He, C. Wang, W. Cai, J. Sui, Z. Ren, Understanding and manipulating the intrinsic point defect in $\alpha-\mathrm{MgAgSb}$ for higher thermoelectric performance, Journal of Materials Chemistry A 4 (2016) 16834-16840.

[45] P. Ying, X. Liu, C. Fu, X. Yue, H. Xie, X. Zhao, W. Zhang, T. Zhu, High performance $\alpha-\mathrm{MgAgSb}$ thermoelectric materials for low temperature power generation, Chemistry of Materials 27(3) (2015) 909-913.

[46] Z. Liu, Y. Wang, W. Gao, J. Mao, H. Geng, J. Shuai, W. Cai, J. Sui, Z. Ren, The influence of doping sites on achieving higher thermoelectric performance for nanostructured $\alpha-\mathrm{MgAgSb}$, Nano Energy 31 (2017) 194-200.

[47] K. Katoh, K. Hisano, T. Takabatake, A. Ochiai, T. Suzuki, Searching and Crystal Growth of $\mathrm{YbTX}(\mathrm{T}=$ transition metal, $\mathrm{X}=\mathrm{Sn}, \mathrm{Sb})$, Meeting of the Physical Society of Japan Sectional Meeting, 1996.

[48] M.J. Kirkham, A.M. dos Santos, C.J. Rawn, E. Lara-Curzio, J.W. Sharp, A.J. Thompson, Abinitio determination of crystal structures of the thermoelectric material MgAgSb, Physical Review B 85(14) (2012) 144120. 
[49] G. Kresse, D. Joubert, FROM ULTRASOFT PSEUDOPOTENTIALS TO THE PROJECTOR AUGMENTED-WAVE METHOD, Physical Review B Condensed Matter 59(3) (1999) 1758-1775.

[50] J.P. Perdew, K. Burke, M. Ernzerhof, Generalized Gradient Approximation Made Simple, Physical Review Letters 78(7) (1997) 1396.

[51] H.J. Monkhorst, Special points for Brillouin-zone integrations, Physical Review B 13(12) (1976) 5188--5192.

[52] A. Togo, I. Tanaka, First principles phonon calculations in materials science, Scripta Materialia 108 (2015) 1-5.

[53] M.T. Dove, Structure and Dynamics - An Atomic View of Materials, Materials Today 6(6) (2003) 59-59.

[54] H.S. Kim, W. Liu, G. Chen, C.-W. Chu, Z. Ren, Relationship between thermoelectric figure of merit and energy conversion efficiency, Proceedings of the National Academy of Sciences 112(27) (2015) 8205-8210.

[55] J. Shen, Z. Chen, L. Zheng, W. Li, Y. Pei, Single parabolic band behavior of thermoelectric p-type $\mathrm{CuGaTe}_{2}$, Journal of Materials Chemistry C 4(1) (2016) 209-214.

[56] H.J. Goldsmid, Introduction to thermoelectricity, Springer Science \& Business Media, 2009.

[57] T.M. Tritt, Thermal conductivity: theory, properties, and applications, Springer Science \& Business Media, 2005.

[58] J. Callaway, Model for lattice thermal conductivity at low temperatures, Physical Review 113(4) (1959) 1046.

[59] J. He, S.N. Girard, M.G. Kanatzidis, V.P. Dravid, Microstructure - Lattice Thermal Conductivity Correlation in Nanostructured $\mathrm{PbTe}_{0.7} \mathrm{~S}_{0.3}$ Thermoelectric Materials, Advanced Functional Materials 20(5) (2010) 764-772.

[60] C. Kittel, Introduction to solid state physics, Wiley, 2005.

[61] E.S. Toberer, A. Zevalkink, G.J. Snyder, Phonon engineering through crystal chemistry, Journal of Materials Chemistry 21(40) (2011) 15843-15852.

[62] J. An, A. Subedi, D.J. Singh, Ab initio phonon dispersions for PbTe, Physics 148(9) (2008) 417-419.

[63] M. Christensen, A.B. Abrahamsen, N.B. Christensen, F. Juranyi, N.H. Andersen, K. Lefmann, J. Andreasson, C.R. Bahl, B.B. Iversen, Avoided crossing of rattler modes in thermoelectric materials, Nature Materials 7(10) (2008) 811-5.

[64] W. Li, J. Carrete, N. A. Katcho, N. Mingo, ShengBTE: A solver of the Boltzmann transport equation for phonons, Computer Physics Communications 185(6) (2014) 1747-1758.

[65] Z. Liu, W. Gao, X. Meng, X. Li, J. Mao, Y. Wang, J. Shuai, W. Cai, Z. Ren, J. Sui, Mechanical properties of nanostructured thermoelectric materials $\alpha-\mathrm{MgAgSb}$, Scripta Materialia 127 (2017) 72-75.

[66] G. Leibfried, E. Schlömann, A. der Wissenschaften, M.-p. Klasse, Wärmeleitung in elektrisch isolierenden Kristallen, von Günther Leibfried und Ernst Schlömann, Vandenhoeck und Ruprecht, 1954. 


\section{Figure Caption}

Figure 1. Synergistic strategies to enhance the thermoelectric performance via $\mathrm{Yb}$ doping. (a) A schematic diagram about the effect of tuning Fermi level, (b) schematic illustration of the microscopic origin of low lattice thermal conductivity for $\mathrm{MgAgSb}$, (c) a schematic diagram about point-defect phonon scattering, (d) ZT dependence of temperature, (e) comparison of average $Z T$ and calculated efficiency for undoped, Li doped [43], and $\mathrm{Yb}$ doped $\mathrm{MgAgSb}$.

Figure 2. Electrical transport properties of $\mathrm{Mg}_{1-\mathrm{x}} \mathrm{Yb}_{\mathrm{x}} \mathrm{Ag}_{0.97} \mathrm{Sb}_{0.99}(\mathrm{x}=0,0.25 \%, 0.5 \%$ and $1 \%$ ). (a) Doping concentration dependent carrier concentration and mobility, (b) temperature dependent electrical resistivity, (c) temperature dependent Seebeck coefficient (d) carrier concentration dependent Seebeck coefficient at room temperature, (e) temperature dependent power factor, (f) carrier concentration dependent power factor. The solid line in (d) and (f) are calculated based on single parabolic band model and acoustic phonon scattering mechanism. The filled diamond, unfilled square, and unfilled circle represent the measurement data for $\mathrm{Yb}$ doping, Li doping [43], and tuning of the Sb content [42], respectively.

Figure 3. (a) Total thermal conductivity $\kappa_{\text {total }}$ and (b) lattice thermal conductivity $\kappa_{\text {lat }}$ dependence of temperature for $\mathrm{Mg}_{1-\mathrm{x}} \mathrm{Yb}_{x} \mathrm{Ag}_{0.97} \mathrm{Sb}_{0.99}(\mathrm{x}=0,0.25 \%, 0.5 \%$ and $1 \%)$.

Figure 4. (a) Phonon dispersions and (b) the corresponding Grüneisen dispersions of MgAgSb. The thick lines and thin lines in the top panel represent the phonon dispersions at the DFT equilibrium volume and the expansion cell $(+4 \%)$, respectively. The red, green, blue and orange lines highlight TA, TA', LA, and LO phonon branches, respectively. The inset is the first Brillouin zones of $\mathrm{MgAgSb}$ with high-symmetry points (red points) that are considered in our current calculations.

Figure 4. Typical microstructures for $\mathrm{Mg}_{0.995} \mathrm{Yb}_{0.005} \mathrm{Ag}_{0.97} \mathrm{Sb}_{0.99}$ sample. (a) SEM 
image, (b) high magnification TEM image showing high density of stacking faults. The inset corresponds to the fast Fourier transformation (FFT) image along the [001] direction, (c) corresponding inverse FFT (IFFT) image of stacking fault, (d) corresponding IFFT image of high density of dislocations along (040) plane. The inset means the geometric phase analysis (GPA) image of dislocation core. 
a

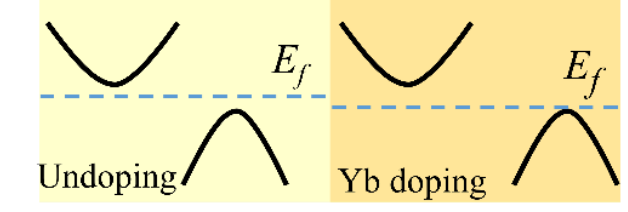

c

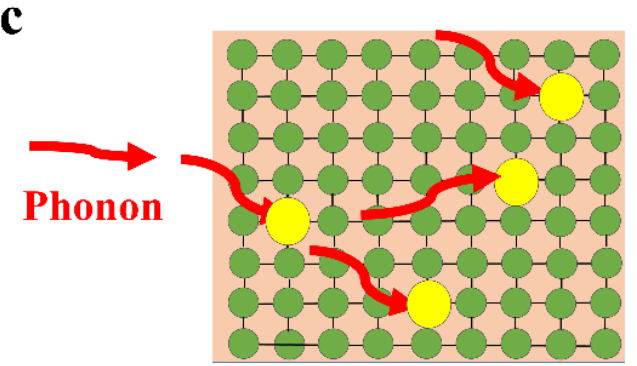

d

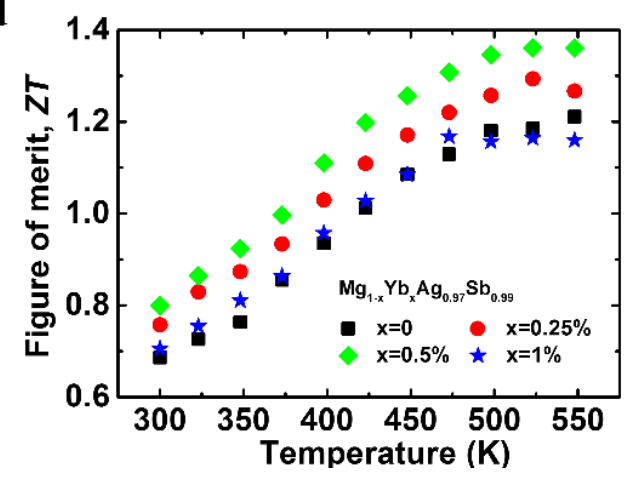

b

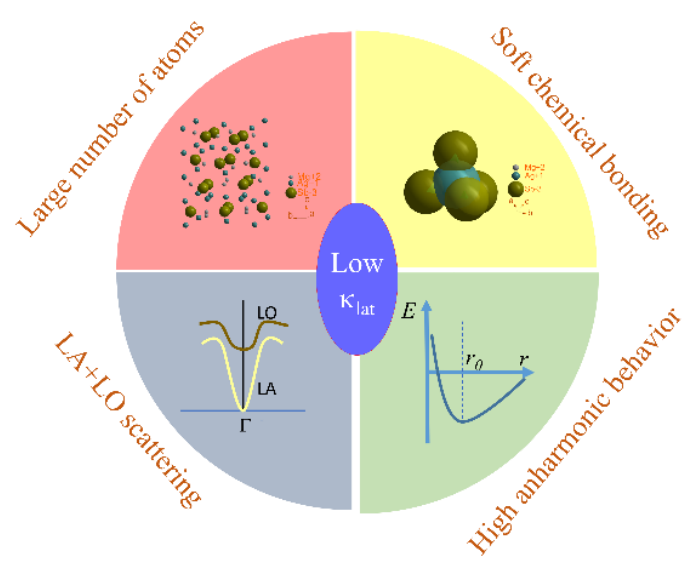

e

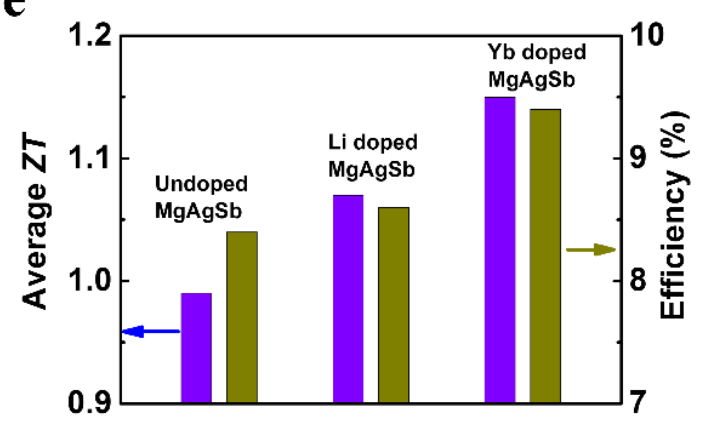

Figure 1 

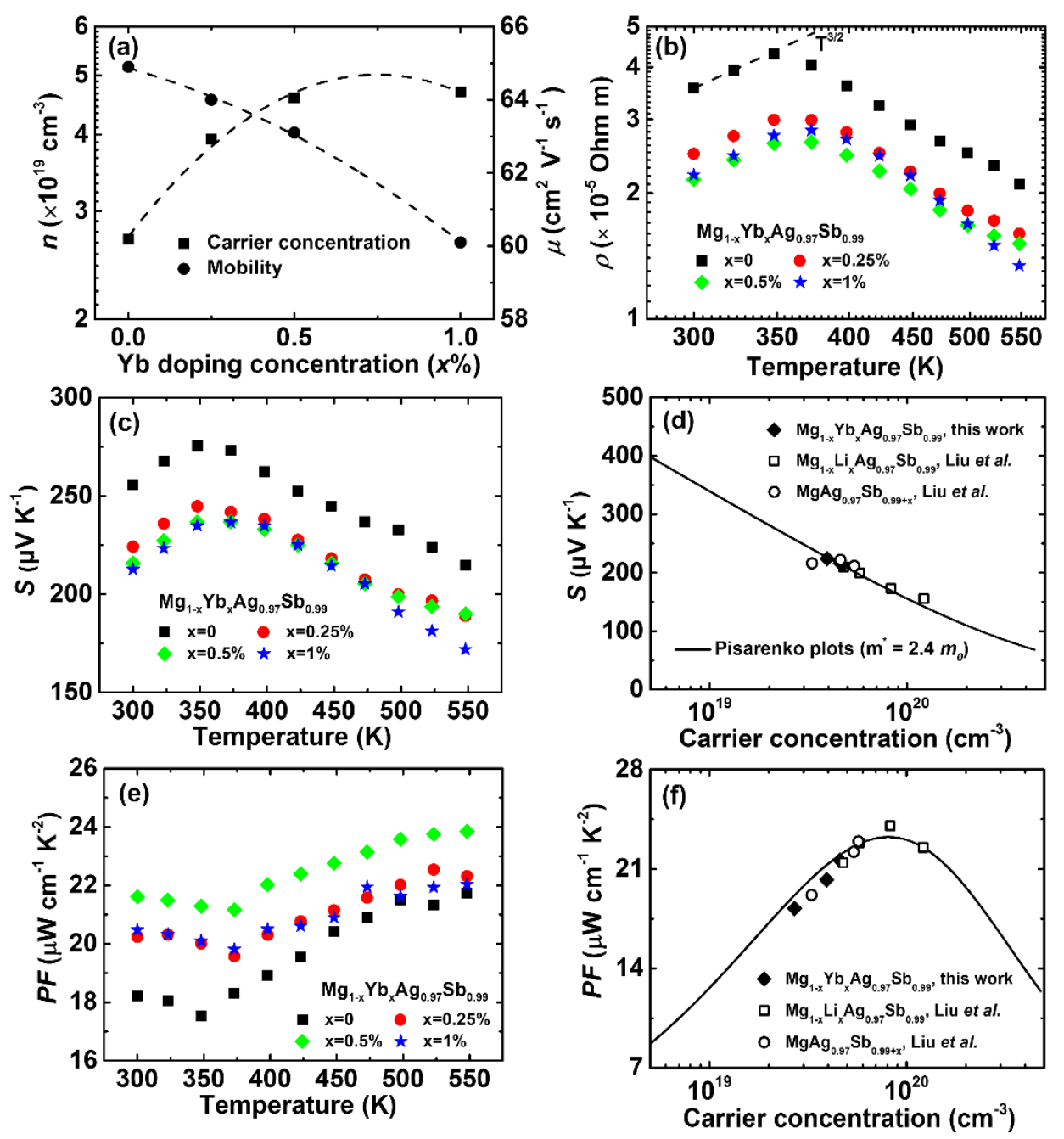

Figure 2 

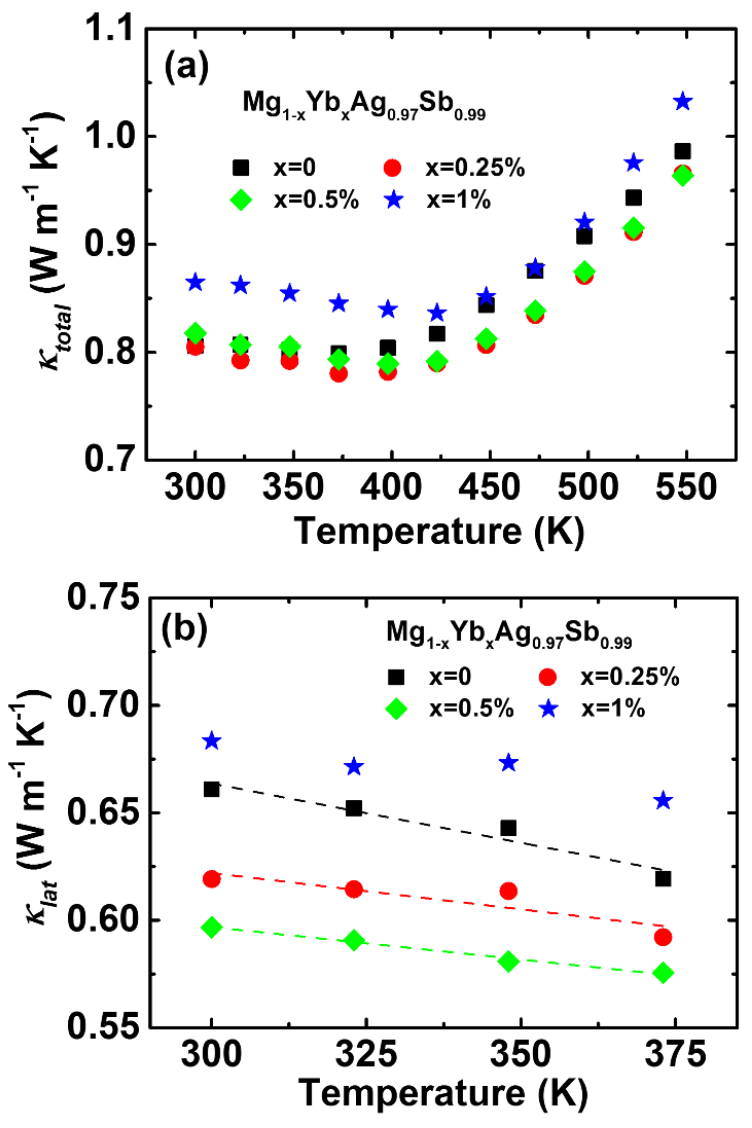

Figure 3 


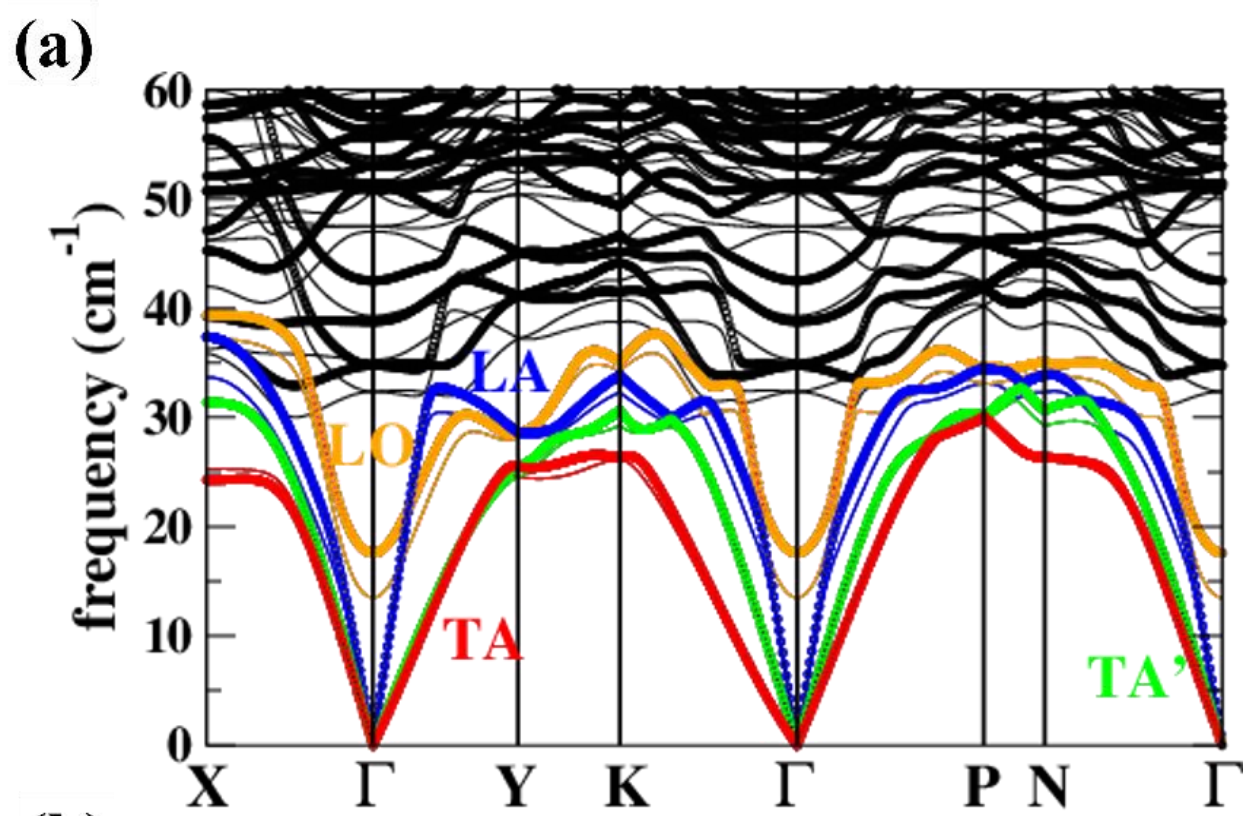

(b)

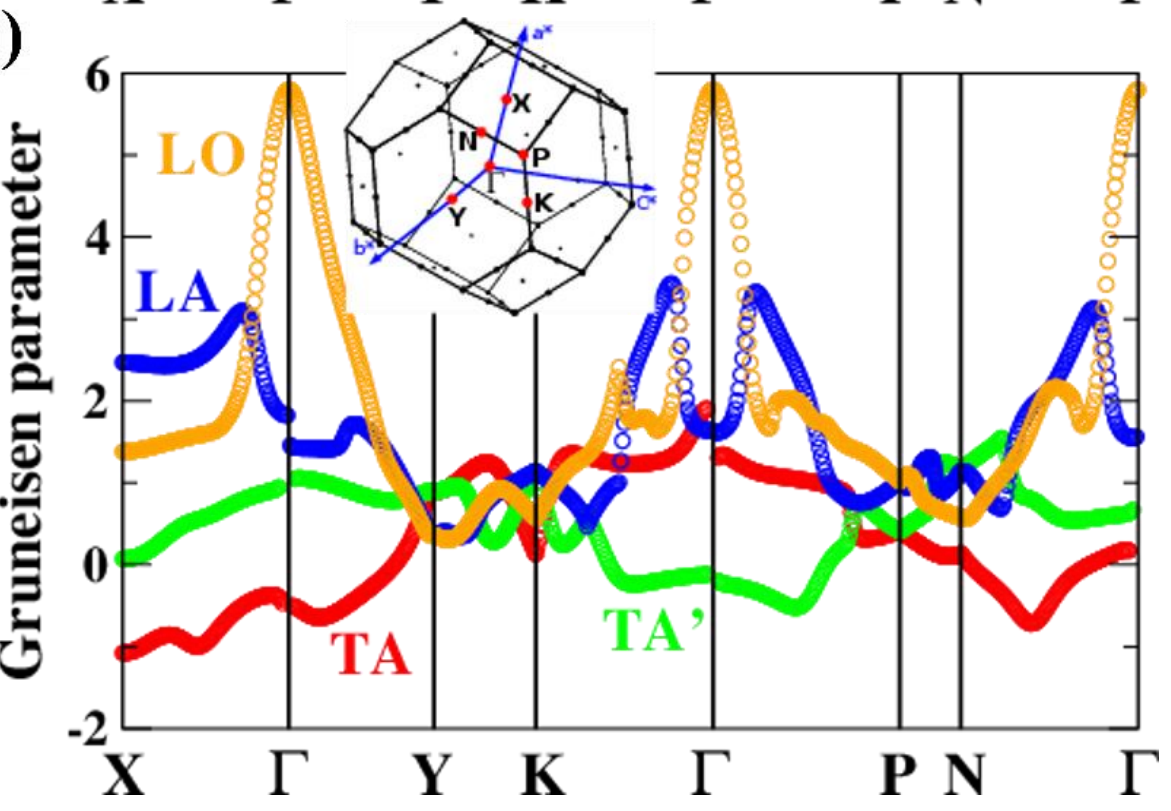

Figure 4 

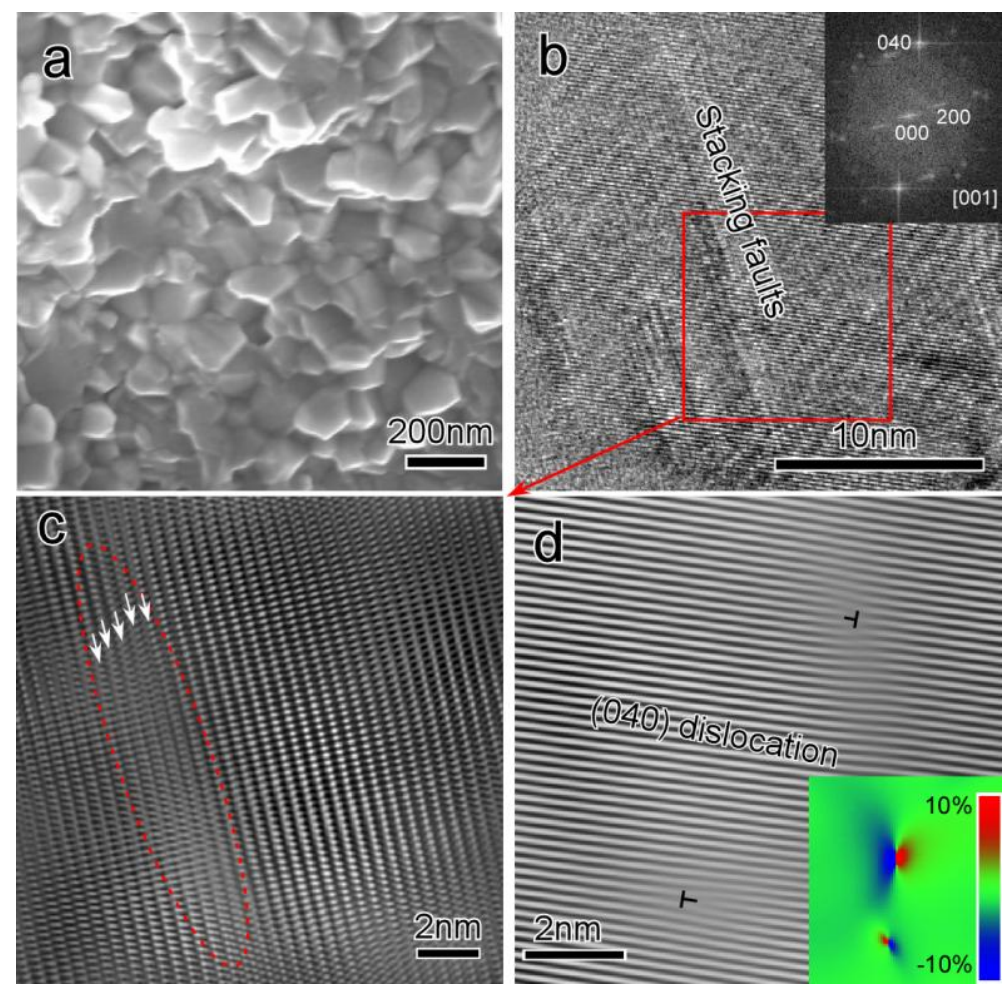

Figure 5 
Table 1. Electric transport properties of $\mathrm{Mg}_{1-\mathrm{x}} \mathrm{Yb}_{\mathrm{x}} \mathrm{Ag}_{0.97} \mathrm{Sb}_{0.99}(\mathrm{x}=0,0.25 \%, 0.5 \%$ and $1 \%)$

\begin{tabular}{ccccc}
\hline Composition & $n_{H}$ & $\mu_{H}$ & $m^{*}$ & $\eta$ \\
& $\left(10^{19} \mathrm{~cm}^{-3}\right)$ & $\left(\mathrm{cm}^{2} \mathrm{~V}^{-1} \mathrm{~s}^{-1}\right)$ & $\left(m_{e}\right)$ & \\
\hline $\mathrm{MgAg}_{0.97} \mathrm{Sb}_{0.99}$ & 2.7 & 64.9 & 2.35 & -0.75 \\
$\mathrm{Mg}_{0.9975} \mathrm{Yb}_{0.0025} \mathrm{Ag}_{0.97} \mathrm{Sb}_{0.99}$ & 3.9 & 64.0 & 2.33 & -0.31 \\
$\mathrm{Mg}_{0.995} \mathrm{Yb}_{0.005} \mathrm{Ag}_{0.97} \mathrm{Sb}_{0.99}$ & 4.6 & 63.1 & 2.39 & -0.18 \\
$\mathrm{Mg}_{0.99} \mathrm{Yb}_{0.01} \mathrm{Ag}_{0.97} \mathrm{Sb}_{0.99}$ & 4.7 & 60.1 & 2.37 & -0.13 \\
\hline
\end{tabular}


Table 2. Fitting parameters for lattice thermal conductivity $\kappa_{l a t}$ in the Debye-Callaway model

\begin{tabular}{cccc}
\hline Composition & $C\left(10^{-18} \mathrm{~s} \mathrm{~K}^{-1}\right)$ & $A\left(10^{-40} \mathrm{~s}^{3}\right)$ & $L_{\text {eff }}(\mathrm{nm})$ \\
\hline $\mathrm{MgAg}_{0.97} \mathrm{Sb}_{0.99}$ & 8.98 & 1.99 & 100 \\
$\mathrm{Mg}_{0.9975} \mathrm{Yb}_{0.0025} \mathrm{Ag}_{0.97} \mathrm{Sb}_{0.99}$ & 8.98 & 2.38 & 100 \\
$\mathrm{Mg}_{0.995} \mathrm{Yb}_{0.005} \mathrm{Ag}_{0.97} \mathrm{Sb}_{0.99}$ & 8.98 & 2.43 & 100 \\
\hline
\end{tabular}


Table 3. Average Debye temperature, phonon velocity, and Grüneisen parameter of longitudinal (LA) and transverse (TA/TA') phonons in MgAgSb calculated from the phonon dispersion

\begin{tabular}{cccc}
\hline Phonon type & $\begin{array}{c}\text { Debye } \\
\text { Temperature }(\mathrm{K})\end{array}$ & $\begin{array}{c}\text { Phonon velocity } \\
\left(\mathrm{m} \mathrm{s}^{-1}\right)\end{array}$ & $\begin{array}{c}\text { Grüneisen } \\
\text { parameter }\end{array}$ \\
\hline TA & 39 & 1488 & 0.8 \\
TA $^{\prime}$ & 44 & 1815 & 0.6 \\
LA & 49 & 3005 & 1.9 \\
\hline
\end{tabular}




\section{Graphical abstract}

The microscopic origin of intrinsically low $\kappa_{\text {lat }}$ of $\alpha-\mathrm{MgAgSb}$ in the lattice dynamics was thoroughly revealed using density functional theory (DFT) calculations. More importantly, we achieve a peak $Z T \sim 1.4$ and average $Z T \sim 1.2$ via $\mathrm{Yb}$ doping.
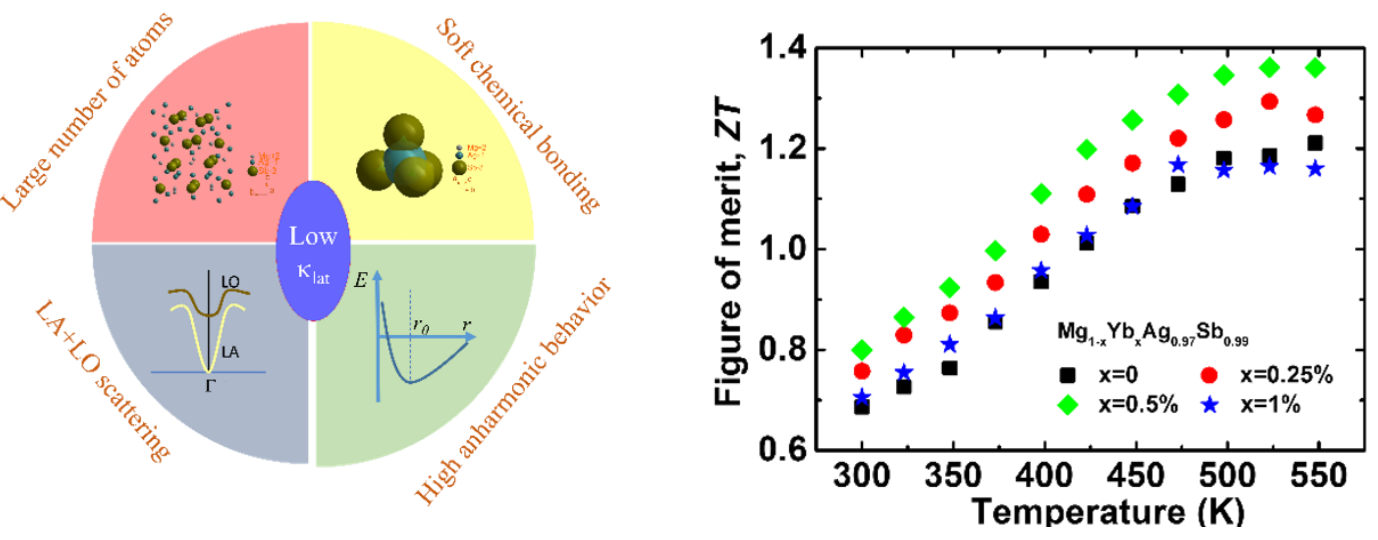